\title{
Surface-active hollow titanosilicate particles as a Pickering interfacial catalyst for liquid-phase alkene epoxidation reactions
}

\author{
Guojun Lv, ${ }^{\mathrm{a}}$ Fumin Wang, ${ }^{\mathrm{a}, *}$ Xubin Zhang ${ }^{\mathrm{a}, *}$ and Bernard P. Binks ${ }^{\mathrm{b}, *}$ \\ ${ }^{a}$ School of Chemical Engineering and Technology, Tianjin University, Tianjin 300350, \\ P.R. China \\ ${ }^{b}$ School of Mathematics and Physical Sciences, University of Hull, \\ Hull. HU6 7RX. U.K. \\ *Corresponding authors: wangfumin@tju.edu.cn (Fumin Wang) \\ tjzxb@tju.edu.cn (Xubin Zhang) \\ b.p.binks@hull.ac.uk (Bernard Paul Binks)
}

Notes

The authors declare no competing financial interest.

Supporting Information

SEM and TEM images of conventional TS-1 zeolite, SEM images of the compressed disks for the HTS-1 particles, digital photographs of W/O emulsions, cryo-SEM image of $\mathrm{W} / \mathrm{O}$ emulsion of cyclohexene + aqueous hydrogen peroxide, catalytic activity and selectivity for the alkene epoxidation, interfacial area and interface coverage with HMDSO/HTS-1 ratio, nitrogen adsorption-desorption isotherms for the modified HTS-1 particles.

This document is the Accepted Manuscript version of a Published Work that appeared in final form in Langmuir, copyright $\odot$ American Chemical Society after peer review and technical editing by the publisher.

To access the final edited and published work see https://pubs.acs.org/doi/10.1021/acs.langmuir.7b03633 


\begin{abstract}
Design of catalyst particles bearing excellent catalytic activity and suitable surface wettability is the key to the successful application of Pickering interfacial catalysis. In this study, the epoxidation of 1-hexene and cyclohexene with aqueous hydrogen peroxide over hollow TS-1 zeolite was studied as probe reactions to investigate the influence of catalyst surface wettability on catalytic activity. Hydrophobized hollow TS-1 particles (HTS-1) were fabricated via a post-synthesis desilication treatment with tetrapropylammonium hydroxide (TPAOH) and a post-synthesis silylation treatment with hexamethyldisiloxane (HMDSO). The successful preparation of HTS-1 particles was verified by scanning electron microscopy (SEM), energy dispersive X-ray spectroscopy (EDS) and Fourier-transform infrared spectroscopy (FTIR). X-ray diffraction patterns and UV-visible spectra confirmed that hydrophobic modification had no effect on the zeolite structure of HTS-1 particles. Stable Pickering emulsions of aqueous hydrogen peroxide in either 1-hexene or cyclohexene could be prepared using HTS-1 particles as emulsifier and confirmed by cryo-SEM images. The catalytic behaviour in the obtained Pickering emulsions revealed a parabolic distribution of turnover frequency (TOF) values with respect to the hydrophobization degree with $0.2-\mathrm{HMDSO} / \mathrm{HTS}-1$ particles possessing the maximum value of $20.6 \mathrm{~h}^{-1}$ for 1 -hexene epoxidation and 8.1 $\mathrm{h}^{-1}$ for cyclohexene epoxidation. In addition, these 0.2-HMDSO/HTS-1 particles showed good re-usability for more than five cycles.
\end{abstract}

Keywords: Pickering interfacial catalysis, alkene epoxidation, hollow TS-1. 


\section{Introduction}

Zeolites, with shape selectivity, high acidity and good hydrothermal stability, are extensively used as heterogeneous catalysts in many industrial processes ${ }^{1,2}$. The titanium-containing silicate named TS-1, first reported by the company Snamprogetti $\mathrm{SpA}$ in 1983 , has gained great interest due to its remarkable catalytic properties in the liquid-phase selective oxidations of aromatic hydroxylation ${ }^{3}$, alkene epoxidation ${ }^{4}$ and ketone ammoximation ${ }^{5,6}$ using hydrogen peroxide as the oxidant under mild conditions. The aforementioned alkene epoxidation reactions are typically liquid-liquid biphasic reactions, i.e. at a planar interface. Organic solvents are generally employed in the chemical synthesis to boost the miscibility of the reagents and promote catalytic activity. For example, methanol is commonly applied as a solvent for 1-hexene epoxidation with hydrogen peroxide over TS-1 ${ }^{7,8}$ whereas acetonitrile is preferred for cyclohexene epoxidation ${ }^{9}$. However, the use of solvents artificially increases the complexity of epoxidation reaction processes which require further purification steps for recycling, affecting negatively the economy and green footprint of these reactions. Accordingly, it would be highly desirable to design solvent-free epoxidation reactions.

In solvent-free biphasic reactions, the main issue is resilient mass/heat transfer ascribed to modest interfacial contact, which always results in low catalytic activity. Pickering interfacial catalysis has recently emerged for boosting the activity of heterogeneous catalysts in the presence of immiscible reagents. Pickering interfacial catalysis $^{10,11}$ is based on the generation of a stable Pickering emulsion between immiscible reagents by the adsorption of colloidal catalyst particles at the oil-water interface. It is expected to open an avenue to the development of biphasic green catalytic systems without addition of solvent, having economic and environmental benefits. This novel methodology combines the advantages of phase transfer and heterogeneous catalysis ${ }^{12-15}$ and has the following attributes: (i) increased interfacial contact area, (ii) enhanced inter-phase mass transfer, (iii) effective separation of final products and (iv) simplified recycling of catalysts.

Pickering interfacial catalysis encompasses particles behaving concomitantly as 
emulsifier and interfacial catalyst, and thus the design of catalyst particles is the key to the successful application of Pickering interfacial catalysis. Ideal catalyst particles suitable as a Pickering emulsifier should be sub-micron and monodisperse and possess a suitable hydrophilic-hydrophobic balance ${ }^{16,17}$. An efficient interfacial catalyst is one allowing easy diffusion to active sites and possesses a large number of active sites. Yang et al. synthesized a partially hydrophobized center-radially fibrous silica encapsulated TS-1 zeolite and successfully used it as a Pickering interfacial catalyst for solvent-free liquid-phase oxidation reactions ${ }^{18}$. However, the center-radially fibrous mesoporous silica is totally inactive for oxidation reactions, which may hinder diffusion of reactants to the TS- 1 core. In addition, the active TS-1 zeolite core is microporous and its bi-directional micropore close to $0.55 \mathrm{~nm}$ strongly hinders the diffusion of reactants to Ti active centers and thus constrains its catalytic activity. The preparation of hollow TS-1 zeolite particles with additional mesopores or macropores ${ }^{19-21}$ can effectively enhance the diffusion and accessibility to Ti-active sites and accordingly improve catalyst catalytic activity. Post-treatment desilication via base leaching ${ }^{22}$ was widely studied in the past decade due to the simple and efficient improvement of the catalytic performance. Here, we first fabricated hollow TS-1 particles via a desilication process with tetrapropylammonium hydroxide (TPAOH) to impart catalytic behaviour. These were then hydrophobically modified with a silane reagent to endow them with suitable surface-active activity. Investigating the relationship between the catalyst catalytic activity and its surface wettability is important in designing eminent TS-1 catalyst particles. Alkene epoxidations with hydrogen peroxide over the TS-1 particles in Pickering emulsions were used as probe reactions to study the influence of catalyst surface wettability on catalytic activity.

\section{Experimental}

\section{Materials}

The reagents used in this work include tetraethyl orthosilicate, TEOS (Shanghai Civi Chemical Technology Co., China), tetrabutyl orthotitanate, TBOT (98\%, 
Shanghai Civi Chemical Technology Co., China), tetrapropylammonium hydroxide, TPAOH (25 wt.\%, Shanghai Civi Chemical Technology Co., China), hydrogen peroxide (30 wt.\%, Shanghai Civi Chemical Technology Co., China), isopropanol (99.5\%, Shanghai Civi Chemical Technology Co., China), toluene (99.5\%, Shanghai Civi Chemical Technology Co., China), hexamethyldisiloxane, HMDSO (99.8\%, Guangfu Fine Chemical Research Institute), 1-hexene (99.5\%, Shanghai Civi Chemical Technology Co., China) and cyclohexene (99.5\%, Shanghai Civi Chemical Technology Co., China). Deionized (DI) water with a resistivity of $18.2 \mathrm{M} \Omega \mathrm{cm}$ is used throughout. All the reagents are used as received without further purification.

\section{Methods}

(a) Synthesis of hollow TS-1 particles

The conventional TS-1 zeolite was hydrothermally synthesized following the procedure described in ref. 19. Briefly, a mixture of $13.4 \mathrm{~g}$ of TEOS, $3.73 \mathrm{~g}$ of isopropanol and $0.55 \mathrm{~g}$ of TBOT was added dropwise into a $32.0 \mathrm{~g}$ aqueous solution of $12 \mathrm{wt} \% \mathrm{TPAOH}$ at room temperature under vigorous stirring. Then the mixture was heated to $363 \mathrm{~K}$ and stirred for $1 \mathrm{~h}$ to remove ethanol and isopropanol, resulting in the formation of a clear dispersion with a molar composition of $1.0 \mathrm{SiO}_{2}: 0.025$ $\mathrm{TiO}_{2}: 0.3$ TPAOH:25 $\mathrm{H}_{2} \mathrm{O}$. The mixture was transferred into a Teflon-lined stainless steel autoclave and subjected to hydrothermal synthesis at $443 \mathrm{~K}$ for 2 days. After cooling to room temperature, the product was recovered by centrifugation, washed with distilled water, dried at $353 \mathrm{~K}$ in a vacuum oven overnight and finally calcined at $823 \mathrm{~K}$ for $6 \mathrm{~h}$. Subsequently, the conventional TS-1 was treated with $0.06 \mathrm{M} \mathrm{TPAOH}$ in a Teflon-lined stainless steel autoclave at $443 \mathrm{~K}$ for 4 days $^{22}$. The obtained hollow TS-1 (HTS-1) particles were separated by centrifugation, washed with distilled water, dried at $353 \mathrm{~K}$ in a vacuum oven overnight and then calcined at $823 \mathrm{~K}$ for $6 \mathrm{~h}$. The prepared HTS-1 particles possess a Si/Ti ratio of 43 inferred by inductively coupled plasma analysis.

(b) Hydrophobic modification of hollow TS-1 particles 
Hydrophobized HTS-1 particles were prepared by a post-synthesis silylation treatment following ref. 23. In a typical synthesis, $4.0 \mathrm{~g}$ of HTS-1 particles were dispersed in $50 \mathrm{ml}$ of toluene. To the suspension, a certain amount of HMDSO (0.4, $0.8,1.2,1.6$ or $2.0 \mathrm{~g}$ ) was added dropwise under stirring and refluxed at $363 \mathrm{~K}$ for $8 \mathrm{~h}$. Then the resultant suspension was centrifuged, and the precipitate was extracted with deionized water to remove un-adsorbed silane followed by drying at $353 \mathrm{~K}$ in a vacuum oven for $12 \mathrm{~h}$. After drying, silylated particles were obtained and denoted as x-HMDSO/HTS-1, where $\mathrm{x}$ represents the weight ratio of HMDSO to HTS-1.

\section{(c) Characterization}

The morphology of the HTS-1 particles was observed using a scanning electron microscopy, SEM (JEOL S-4800). Transmission electron microscopy (TEM) images were generated with a JEOL JEM-2100 high-resolution instrument. The particle size distribution of HTS-1 particles was determined with a Malvern Mastersizer 2000 instrument. X-ray diffraction (XRD) patterns were recorded on a Bruker D8 Advance $\mathrm{X}$-ray diffractometer using $\mathrm{Cu}-\mathrm{K}_{\alpha}$ radiation with a voltage of $40 \mathrm{kV}$ and a current of $40 \mathrm{~mA}$. Diffractograms were obtained with $2 \theta$ from $10^{\circ}$ to $80^{\circ}$ and a scanning rate of $8 \%$ min. UV-vis diffuse reflection spectroscopy was measured with a Jasco UV 550 $\mathrm{UV}$-vis absorption spectrometer using $\mathrm{BaSO}_{4}$ as a reference to access the nature of $\mathrm{Ti}$ species in the different samples. The conductivities of emulsions were measured immediately using a DDS-307 conductivity meter. IR spectra were monitored using a Tensor 27 Fourier transform infrared (FTIR) spectrometer. The software collected spectra in absorbance mode (wavenumbers from 4000 to $400 \mathrm{~cm}^{-1}$ ). The specific surface area, pore volume and pore size distribution of the HTS-1 samples were measured from $\mathrm{N}_{2}$ adsorption/desorption isotherms at $77 \mathrm{~K}$ on an Micromeritics ASAP 2020 porosimiter. The specific surface area was evaluated using the Brunauer-Emmett-Teller (BET) method, and the micropore volume was calculated by the t-plot method. The total pore volume was measured from the adsorption pore volume at $\mathrm{P} / \mathrm{P}_{0}=0.990$. Moreover, the mesopore size distribution was calculated from the adsorption branch of the isotherm by the Barrett-Joyner-Halenda (BJH) model. 
The chemical compositions of the HTS-1 samples were determined using a Shimadzu ICPS-8000E inductively coupled plasma atomic emission spectrometer. The apparent contact angle $\theta$ of a water drop under oil resting on a solid substrate composed of HTS-1 particles was measured using an optical measuring device (JC2000C1, Shanghai Powereach Co.). The HTS-1 particle powder was compressed into circular disks of thickness $1 \mathrm{~mm}$ using a HY-12 tablet machine under a pressure $18 \mathrm{MPa}$. The disk was carefully placed at the bottom of a transparent vessel which was then half filled with 1-hexene. Then a drop of water was placed on the compressed disk under 1-hexene via a syringe pump. The appearance of the water drop was recorded by a CCD camera, and commercial software was applied to digitalize the image to determine the profile of the water droplet as well as the three-phase contact point. Finally, the apparent contact angle was determined as the angle between the tangent to the three-phase point and the baseline of the disk.

\section{(d) Preparation of Pickering emulsions}

Pickering emulsions of 1-hexene-aqueous hydrogen peroxide (30 wt.\%) and cyclohexene-aqueous hydrogen peroxide (30 wt.\%) stabilized by HTS-1 zeolite particles were prepared according to the following method. $0.2 \mathrm{~g}$ of HTS- 1 particles were weighed in a $50 \mathrm{ml}$ glass beaker at $25^{\circ} \mathrm{C}$ and then dispersed into $10 \mathrm{ml}$ of 1-hexene or cyclohexene using a magnetic stirrer. Next, $10 \mathrm{ml}$ of aqueous hydrogen peroxide was added into the mixture to form HTS-1 stabilized Pickering emulsions (water-in-oil, W/O) at a stirring rate of 10,000 rpm for $10 \mathrm{~min}$. Emulsion droplet morphologies were observed by a high speed CCD camera (AOS X-PRI) and emulsion droplet sizes were determined by light diffraction using a Malvern Mastersizer 2000 instrument.

\section{(e) Catalytic reactions}

Epoxidation reactions were carried out in the obtained stable aqueous hydrogen peroxide-in-1-hexene and aqueous hydrogen peroxide-in-cyclohexene Pickering emulsions. The reactions were carried out at $333 \mathrm{~K}$ for $2 \mathrm{~h}$ without stirring. After the 
reaction, catalyst particles were removed by centrifugation and the reaction products were analyzed and quantified by gas chromatography (GC-2014C, Shimadzu) using an OV-17 column and a flame ionization detector.

\section{Results and discussion}

\section{(a) Characterization of HTS-1 particles}

The SEM image shown in Figure S1 demonstrates that the prepared conventional TS-1 zeolite has a smooth crystal surface and a regular hexagonal morphology with an average size of about $250-300 \mathrm{~nm}$, which agrees with the high magnification TEM image displayed in Figure S2. When conventional TS-1 particles were treated with $\mathrm{TPAOH}$, the erosion mainly occurred within the interior of the particles, and the dissolved species could be recrystallized ${ }^{24}$. Then, HTS-1 particles with abundant intra-crystalline voids (see Figure 1a) are obtained. The hollow cavities ranging in size from several to tens of nanometers benefits diffusion and accessibility to active sites, giving rise to prominent catalytic activity. The average diameter of the HTS-1 particles is about $300 \mathrm{~nm}$ deduced from the high magnification SEM image shown in Figure 1b, in agreement with the particle size measurement displayed in the inset. Line-scan analyses displayed in Figure 1c confirm successful preparation of HTS-1 particles and homogeneous distribution of $\mathrm{Si}$ and $\mathrm{Ti}$ on their surfaces. The X-ray photoelectron spectrum of Ti2 plotted in Figure 1d demonstrates two Ti2 $\mathrm{p}_{3 / 2}$ characteristic peaks at around $460.7 \mathrm{eV}$ and $459.3 \mathrm{eV}$, which are attributed to tetrahedral and extra-framework $\mathrm{Ti}^{25,26}$ respectively, and two Ti2 $\mathrm{p}_{1 / 2}$ characteristic peaks centered at around $465.2 \mathrm{eV}$ and $466.8 \mathrm{eV}$. 

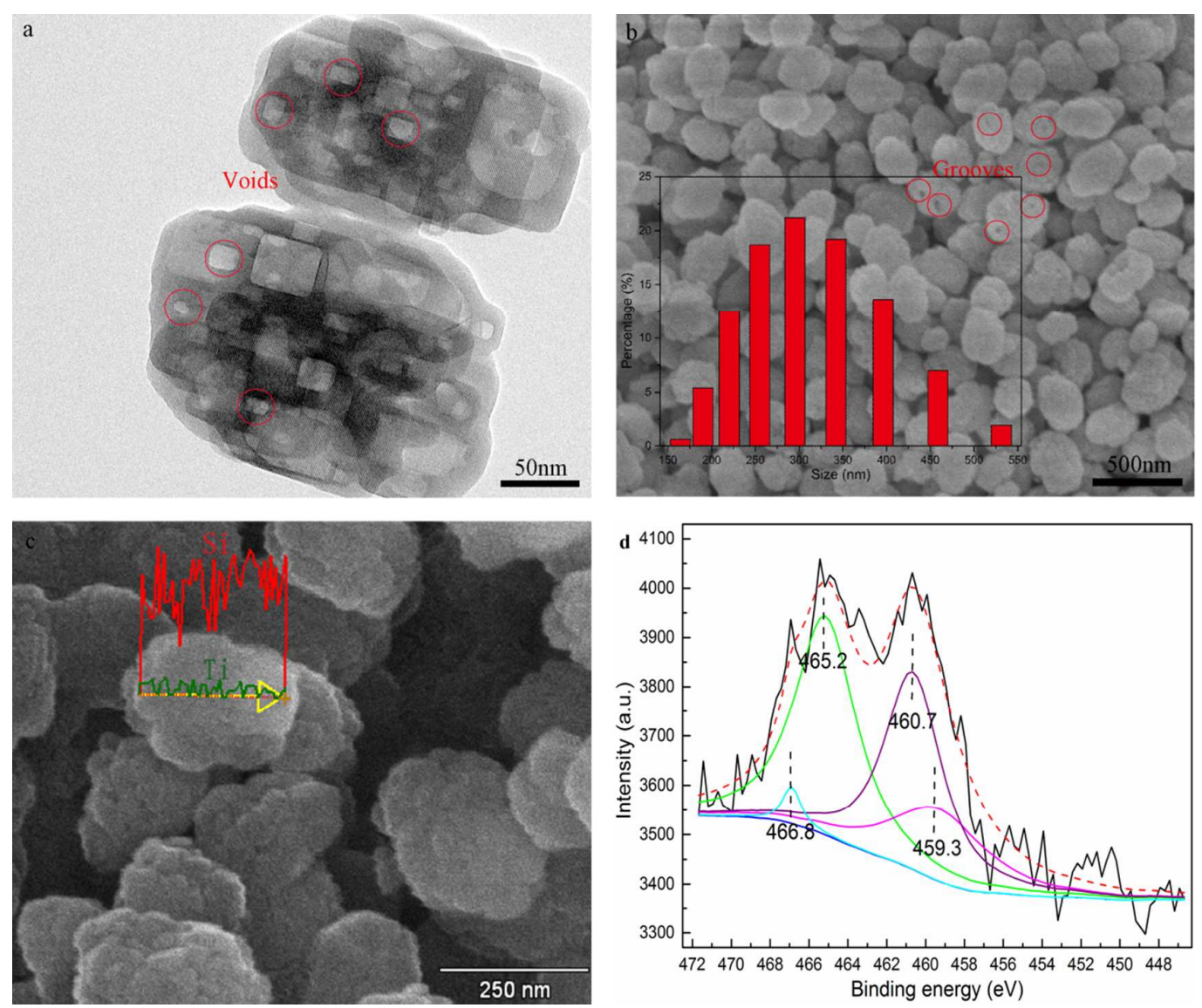

Figure 1. Pristine HTS-1 particles. (a) TEM image, (b) SEM image with size distribution in the inset, (c) line-scan image for elemental $\mathrm{Si}$ and $\mathrm{Ti}$ and (d) high-resolution Ti 2p XPS spectrum.

The nitrogen adsorption/desorption isotherm of the HTS-1 particles is shown in Figure 2. The type IV isotherm with a $\mathrm{H} 4$ hysteresis loop at $\mathrm{P} / \mathrm{P}_{0}>0.45$ caused by capillary condensation reveals that the particles are typically mesoporous. In particular, mesopores centered around $30 \mathrm{~nm}$ were clearly exhibited in the pore size distribution curve. Furthermore, the specific surface area calculated by the Brunauer-Emmet-Teller (BET) model is $437 \mathrm{~m}^{2} / \mathrm{g}$, including a micropore area of 257 $\mathrm{m}^{2} / \mathrm{g}$ and a mesopore area of $180 \mathrm{~m}^{2} / \mathrm{g}$. The average pore size calculated by the Barrent-Joyner-Halenda (BJH) model is $11.8 \mathrm{~nm}$ and the total pore volume is 0.44 $\mathrm{cm}^{3} / \mathrm{g}$ comprised of a micropore volume of $0.12 \mathrm{~cm}^{3} / \mathrm{g}$ and a mesopore volume of 
$0.32 \mathrm{~cm}^{3} / \mathrm{g}$.

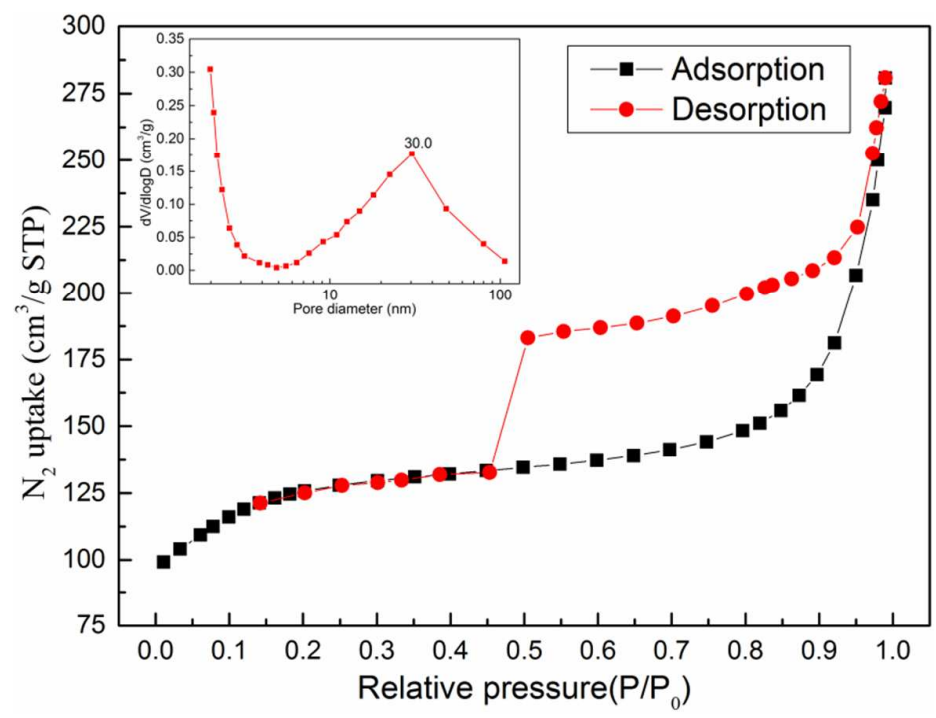

Figure 2. Nitrogen adsorption-desorption isotherm for pristine HTS-1 particles and their pore size distribution in the inset.

\section{(b) Surface modification of HTS-1 particles by silylation}

The size and shape of the HTS-1 zeolite is practically unchanged after modification using silane. As an example, line-scan analyses shown in Figure 3a reveal elemental $\mathrm{C}$ distributed on the partially hydrophobized particles of 0.5-HMDSO/HTS-1, confirming successful anchoring of the trimethylsilyl group, -Si $\left(\mathrm{CH}_{3}\right)_{3}$, on particles. The X-ray photoelectron spectrum of the particles displays similar Ti2p characteristic peaks as with the pristine HTS-1 particles but with an additional characteristic peak at around $284.6 \mathrm{eV}$ (see Figure $3 \mathrm{~b}$ ) ascribed to $\mathrm{C} 1 \mathrm{~s}$, which confirms the successful silanisation modification. At the same time, mapping analyses shown in Figure 3c and d display a homogeneous distribution of elemental C on the hydrophobized HTS-1 particles. 

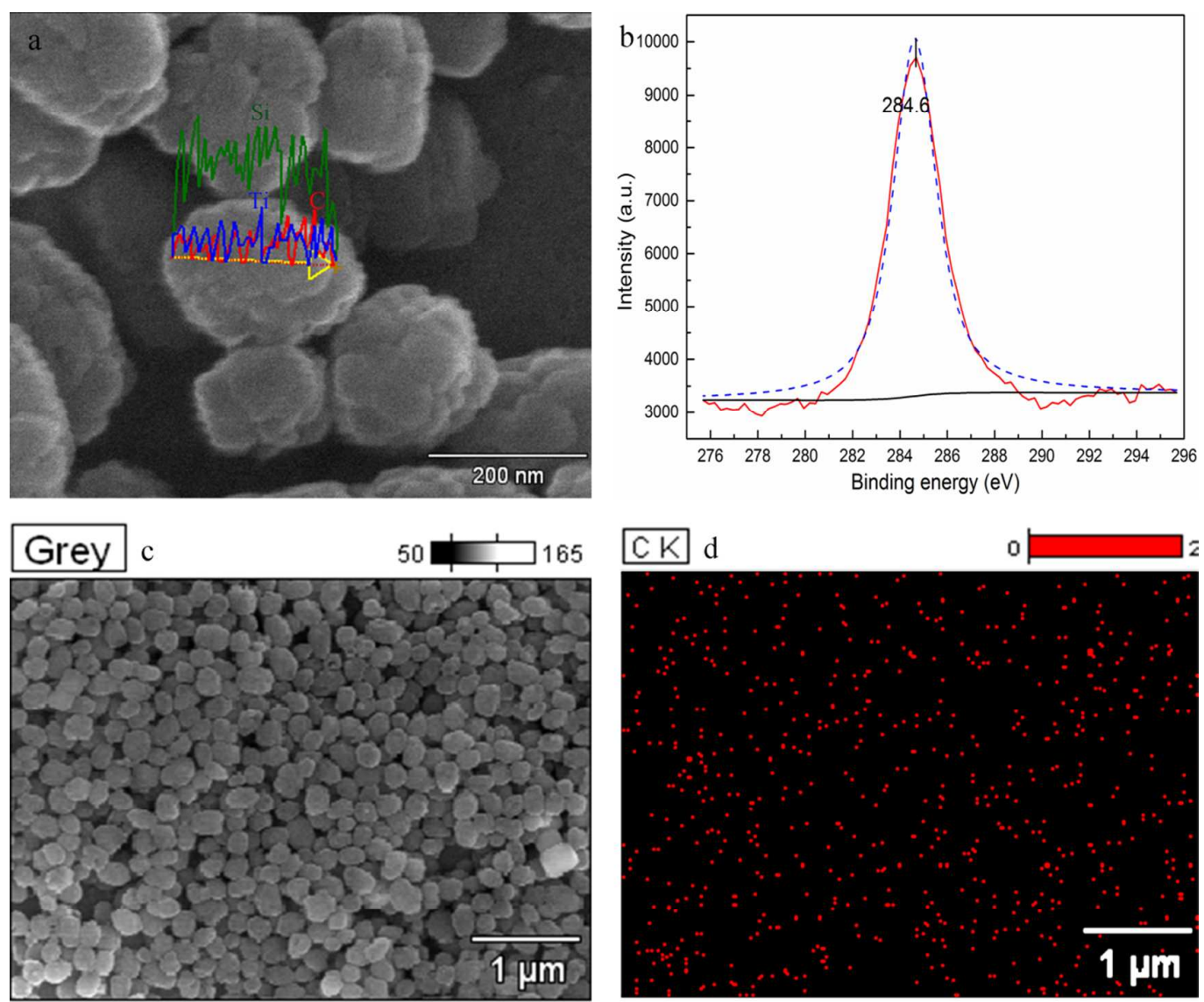

Figure 3. 0.5-HMDSO/HTS-1 particles. (a) Line-scan image for $\mathrm{Si}$, Ti and $\mathrm{C}$, (b) high-resolution C 1s XPS spectrum, (c), (d) mapping image of elemental C.

The X-ray diffraction patterns of the hydrophobized HTS-1 particles for different extents of silanisation are depicted in Figure 4. Notably, they all exhibit similar characteristic peaks at $2 \theta=7.9^{\circ}, 8.8^{\circ}, 23.1^{\circ}, 23.9^{\circ}$ and $24.4^{\circ}$, which correspond to the powder pattern of crystalline MFI zeolite ${ }^{27,28}$, indicating silylation has no obvious effect on the zeolitic topology of HTS-1 particles. 


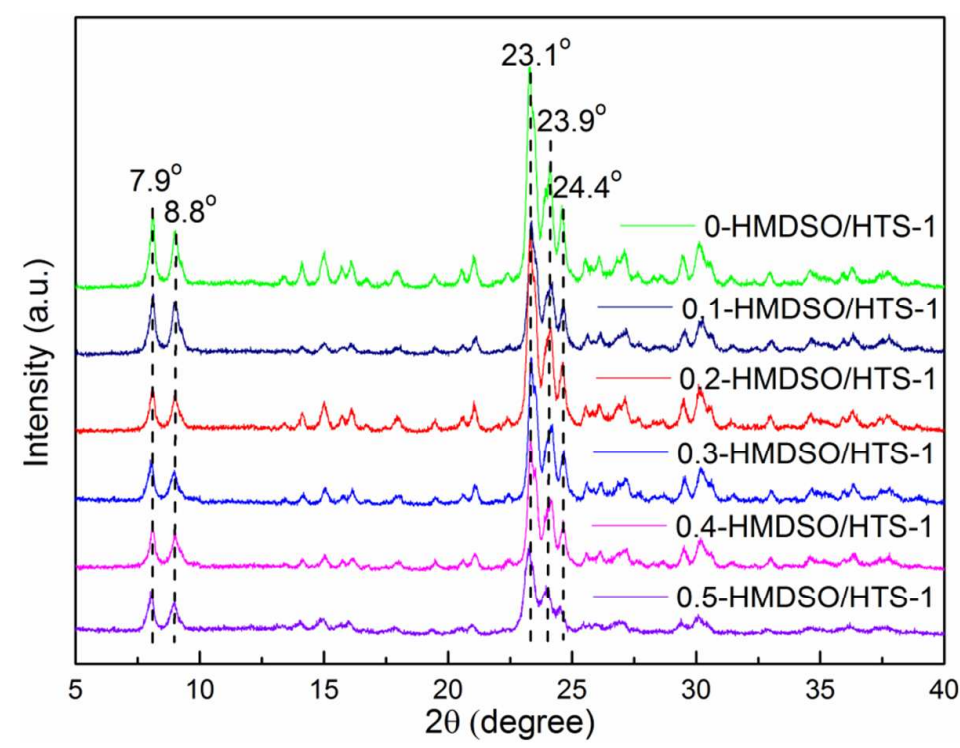

Figure 4. X-ray diffraction patterns of $\mathrm{x}-\mathrm{HMDSO} / \mathrm{HTS}-1$ particles for $\mathrm{x}=0,0.1,0.2$, $0.3,0.4$ and 0.5 .

UV-Visible spectroscopy has been extensively applied to characterize the nature and coordination of titanium atoms in titanium-substituted molecular sieves. Thus, UV spectra of the particles between 200 and $450 \mathrm{~nm}$ were measured and are given in Figure 5. For all the HTS-1 particles, the spectra are dominated by a characteristic band at $218 \mathrm{~nm}$ originating from charge transfer of an oxygen $2 p$ electron to an empty $3 \mathrm{~d}$ orbit of framework Ti species in tetrahedral coordination ${ }^{29}$, demonstrating that the Ti environment remains unaltered by the silylation process. The shoulder band around $265 \mathrm{~nm}$ is ascribed to penta- or even hexa-coordinated titanium resulting from a gradual reduction in adsorbed water molecules upon increasing the silanisation degree $(\mathrm{x})$. 


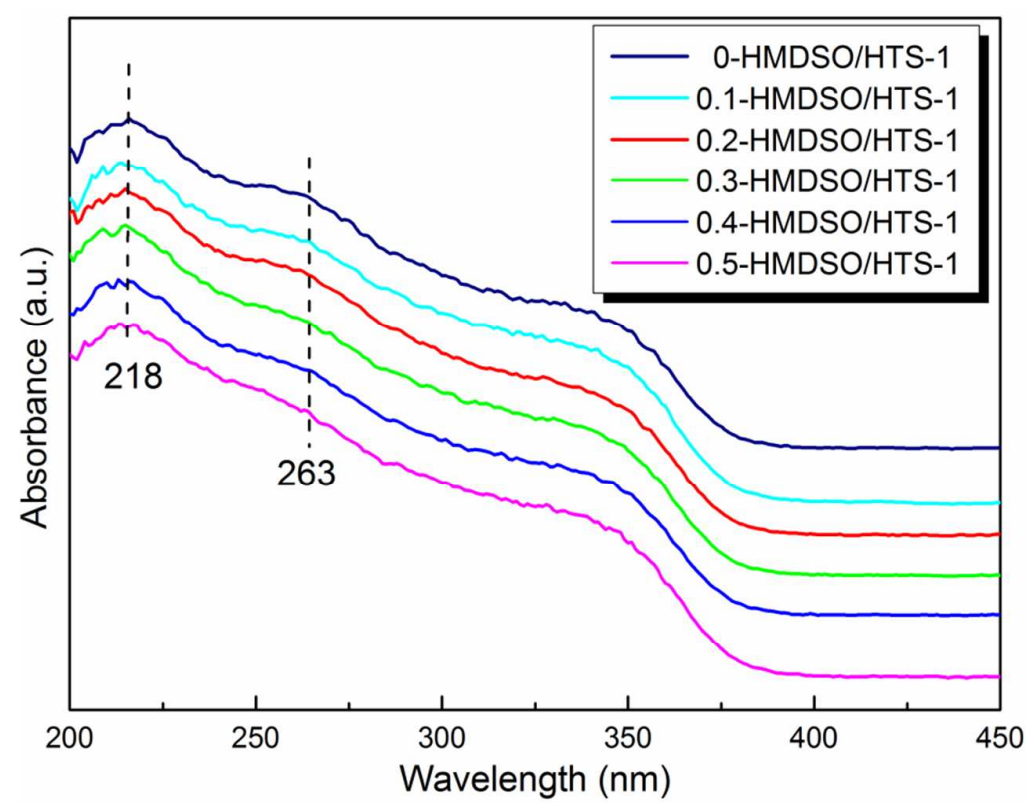

Figure 5. UV-vis spectra of $x-H M D S O / H T S-1$ particles.

Furthermore, IR spectra of the x-HMDSO/HTS-1 samples are included in Figure 6. The spectra display almost the same absorption bands around $450 \mathrm{~cm}^{-1}, 550 \mathrm{~cm}^{-1}$, $800 \mathrm{~cm}^{-1}, 970 \mathrm{~cm}^{-1}, 1100 \mathrm{~cm}^{-1}$ and $1230 \mathrm{~cm}^{-1}$. The band at $550 \mathrm{~cm}^{-1}$ belongs to the characteristic band of the MFI structure ascribed to the double five-membered rings vibration of $\mathrm{SiO}_{4}$ and $\mathrm{TiO}_{4}{ }^{30}$. The band at $970 \mathrm{~cm}^{-1}$ is attributed to the collective vibration of the Si-O-Ti bond influenced by the Ti framework of TS- $1^{31}$. It indicates that the MFI topological structure is stable after the silylation process. Compared to pristine HTS-1 particles (0-HMDSO/HTS-1), the modified HTS-1 particles possess obvious absorption peaks at $2850 \mathrm{~cm}^{-1}$ and $2930 \mathrm{~cm}^{-1}$, which can be assigned to the stretching vibration and bending vibration of the alkyl group $\left(\mathrm{CH}_{3}\right)$ after silanisation. Upon increasing the extent of silanisation (x), the intensities of the alkyl group absorption peaks progressively increase. 


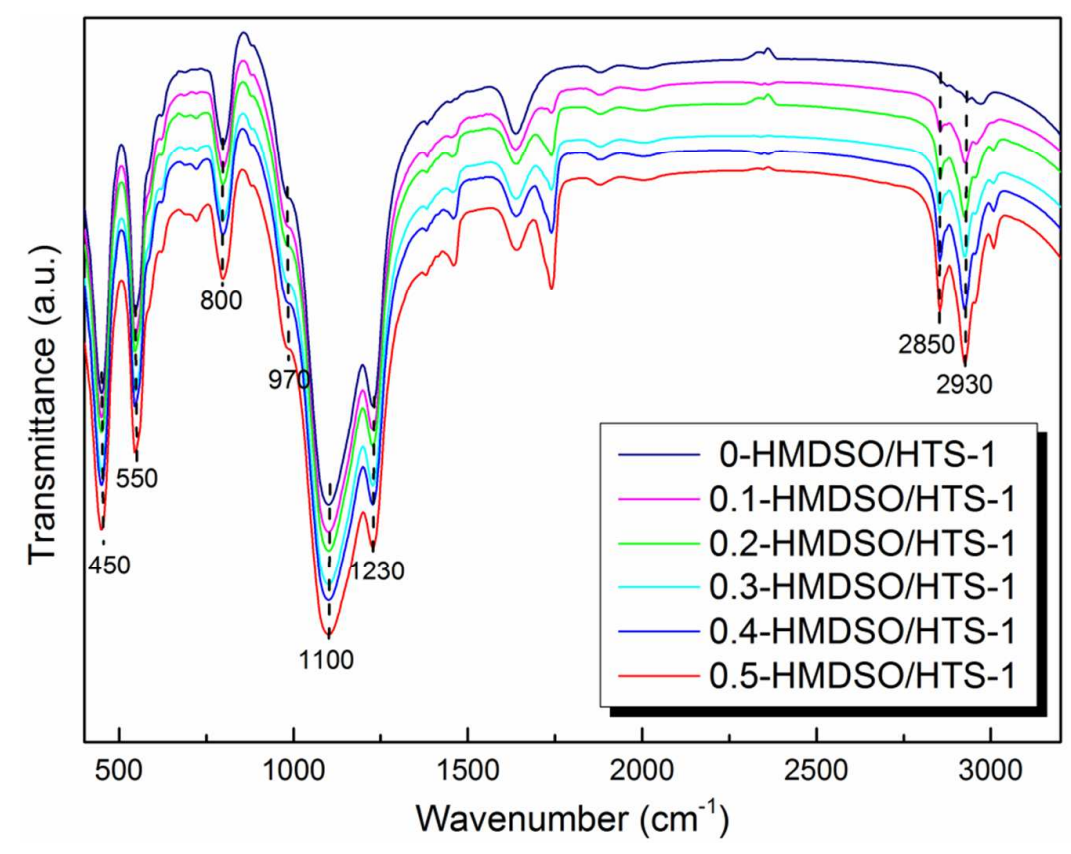

Figure 6. Infrared spectra of x-HMDSO/HTS-1 particles.

\section{(c) Pickering emulsions stabilized by $x$-HMDSO/HTS-1 particles}

Emulsions stabilized solely by colloidal particles have been known for a long time $^{32}$ and the wettability of particles is crucial in dictating their type and stability ${ }^{33}$. Particle wettability may be quantified by the apparent contact angle the particle makes with oil-water interface ${ }^{34,35}$. For a preferentially water wetted particle, the apparent contact angle through water is smaller than $90^{\circ}$ and oil-in-water $(\mathrm{O} / \mathrm{W})$ emulsions are preferred, whereas for hydrophobic particles with an apparent contact angle higher than $90^{\circ}$ water-in-oil (W/O) emulsions are preferred. The x-HMDSO/HTS-1 particle powders were first compressed into relatively flat disks as observed in Figure S3 and then drops of water were placed on the compressed disks under 1-hexene via a syringe pump. The appearances of water droplets on disks were recorded and are shown in Figure 7. Pristine HTS-1 particles possess an apparent contact angle of $45 \pm 2^{\circ}$ whereas apparent contact angles for the hydrophobized HTS-1 samples are all greater than $90^{\circ}$. The apparent contact angles increase from $93 \pm 3^{\circ}$ to $135 \pm 2^{\circ}$ on increasing the degree of silylation $\mathrm{x}$ from 0.1 to 0.5 . 


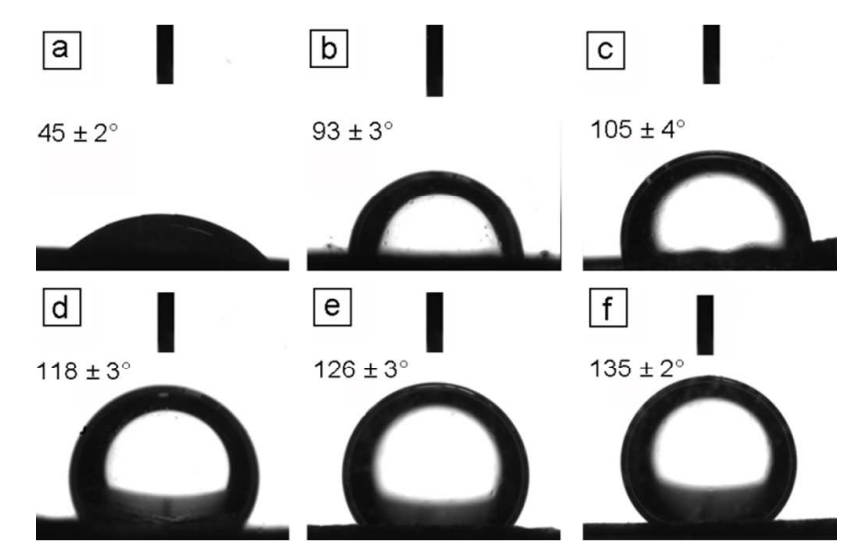

Figure 7. Appearance of water droplets under 1-hexene on compressed particle disks of $\mathrm{x}-\mathrm{HMDSO} / \mathrm{HTS}-1$ for $\mathrm{x}=$ (a) 0 , (b) 0.1 , (c) 0.2 , (d) 0.3 , (e) 0.4 and (f) 0.5 .

The $\mathrm{x}$-HMDSO/HTS-1 particles were used as emulsifiers for the stabilization of 1-hexene + aqueous hydrogen peroxide and cyclohexene + aqueous hydrogen peroxide Pickering emulsions. For the pristine HTS-1 particles (0-HMDSO/HTS-1), an $\mathrm{O} / \mathrm{W}$ emulsion forms due to the hydrophilic nature of the particles. However, it rapidly coalesced in $5 \mathrm{~min}$ with the particles returning to the water phase after complete phase separation. For the modified HTS-1 particles, the obtained emulsions all had conductivities $<1 \mu \mathrm{S} / \mathrm{cm}$ indicating a continuous oil phase. The $\mathrm{W} / \mathrm{O}$ emulsions with 1-hexene were stored in glass vessels to monitor their stability (see Figure S4). All emulsions are stable to coalescence for 4 days with no water being released below the emulsion. After $2 \mathrm{hr}$, emulsions stabilised by particles with $\mathrm{x}=0.1$ to 0.3 show signs of sedimentation releasing an upper oil phase. The extent of sedimentation decreases with increasing particle hydrophobicity. Emulsions stabilised by particles with $\mathrm{x}=0.4$ or 0.5 are stable to sedimentation. The interesting phenomenon that the sedimentation extent decreases with the increase of particle hydrophobicity was also observed in the W/O emulsions for cyclohexene with $\mathrm{x}=0.1$ to 0.4 (see Figure S5). For cyclohexene, emulsions stabilised by particles with $\mathrm{x}=0.5$ are stable to sedimentation. 
Cryo-SEM was employed for further characterization of the W/O emulsion using 1-hexene and cyclohexene stabilised by 0.1-HMDSO/HTS-1 particles. A water droplet in 1-hexene can be seen in Figure $8 \mathrm{a}$ exhibiting surface structure. The magnified image of part of its surface shown in Figure $8 \mathrm{~b}$ clearly shows the 0.1-HMDSO/HTS-1 particles at the interface responsible for an emulsion stable to coalescence. Figures S6a and b also confirm that 0.1-HMDSO/HTS-1 particles predominantly exist at the hydrogen peroxide-cyclohexene interface and stabilize emulsion droplets. The formation of stable microdroplets results in a large increase in oil-water contact area and promotes inter-phase mass transfer and catalytic reactivity ${ }^{36}$.
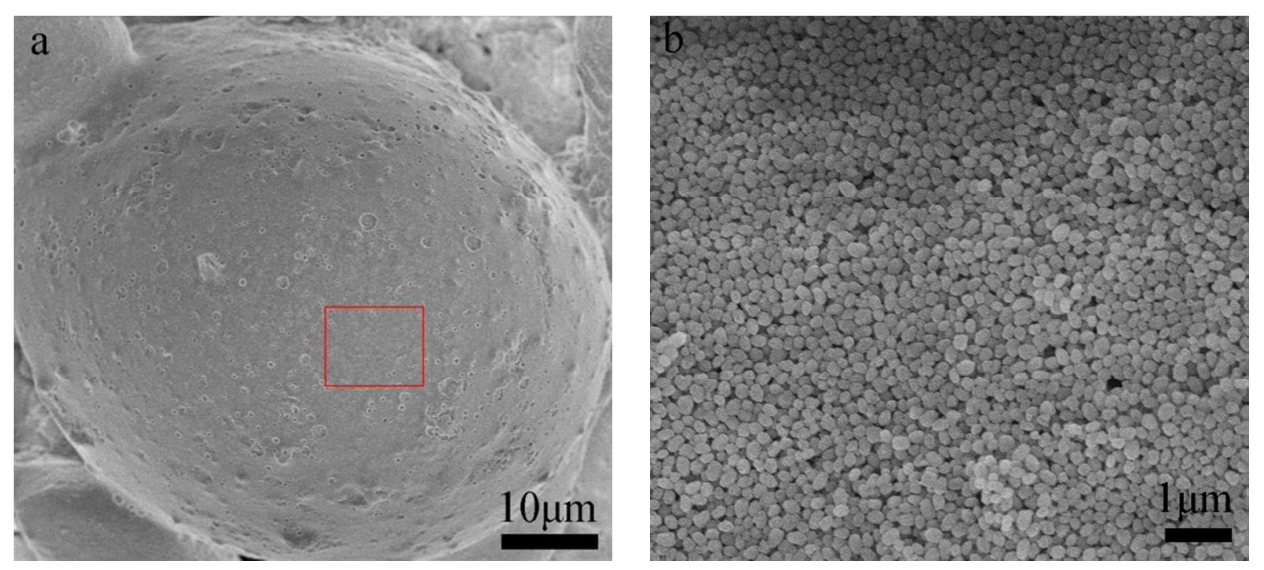

Figure 8. (a) Cryo-SEM image of W/O emulsion of 1-hexene + aqueous hydrogen peroxide stabilized by 0.1 -HMDSO/HTS-1 particles and (b) magnified image of part of its surface.

\section{(d) 1-Hexene and cyclohexene epoxidation in Pickering emulsions}

1-hexene and cyclohexene epoxidation was used to evaluate the catalytic activity of the HTS-1 particles and determine the specific activity per Ti site. Table S1 presents the epoxidation performance of the HTS-1 catalyst for both oils. It is well known that tetrahedral framework Ti species are the main active sites for epoxidation reactions and the amount of this species is closely related to epoxidation results. 
Hence, the obtained low 1-hexene and cyclohexene conversions in the Pickering emulsions may be due to the low amount of Ti active sites. In order to eliminate the effect of Ti amount on catalyst catalytic activity, we use the turnover frequency (TOF) to evaluate the catalytic activity and the corresponding TOF values for the HTS-1 particles are displayed in Figure 9. The TOF values for 1-hexene epoxidation are all larger than those for cyclohexene epoxidation, which may be due to the larger size of cyclohexene molecules and higher diffusion resistance ${ }^{26}$. In 1-hexene epoxidation, the TOF of pristine HTS-1 particles is only $4.5 \mathrm{~h}^{-1}$, lower than the values obtained for all the modified HTS-1 particles. The same applies to the epoxidation of cyclohexene. We attribute this enhanced catalytic behavior with modified HTS-1 particles to the formation of homogeneous W/O Pickering emulsions stabilised by such particles remaining stable for more than $1 \mathrm{~h}$. For 1-hexene epoxidation, 0.1-HMDSO/HTS-1 particles are catalytically active giving rise to a TOF value of $17.7 \mathrm{~h}^{-1}$. The TOF value for $0.2-\mathrm{HMDSO} / \mathrm{HTS}-1$ particles is increased further to $20.6 \mathrm{~h}^{-1}$. Subsequently, the TOF value gradually decreases with the degree of hydrophobization to $16.4,11.4$ and $7.4 \mathrm{~h}^{-1}$ for particles of 0.3-HMDSO/HTS-1, 0.4-HMDSO/HTS-1 and 0.5-HMDSO/HTS-1 respectively. Interestingly, a similar maximum in the TOF value with the hydrophobization degree is also observed for cyclohexene epoxidation. It is apparent that catalyst catalytic activity is closely related to particle wettability and inter-phase mass transfer. 
2

3

4

5

6

7

8

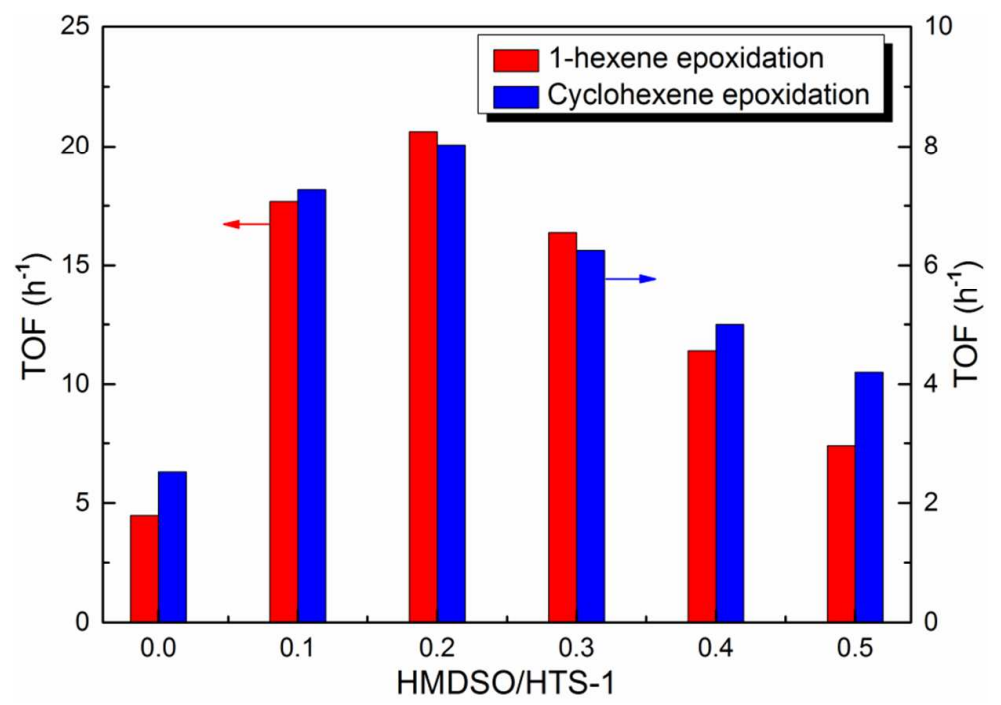

Figure 9. Turnover frequency $(\mathrm{TOF}=$ moles of converted substrate $/$ moles of $\mathrm{Ti}$ species/h) for different HTS-1 particles during the epoxidation of 1-hexene or cyclohexene.

For the case of a stable Pickering emulsion, the phenomena involved during inter-phase mass transfer are rather complex and therefore it is simply described here (see Figure 10). Mass transfer phenomena can mainly be divided into three parts: (i) external diffusion of reactants (dispersed and continuous phase) to outer surface of catalyst particles, (ii) pore diffusion of reactants to active sites of catalyst particles, (iii) interfacial reaction of reactants $\mathrm{s}^{37}$.

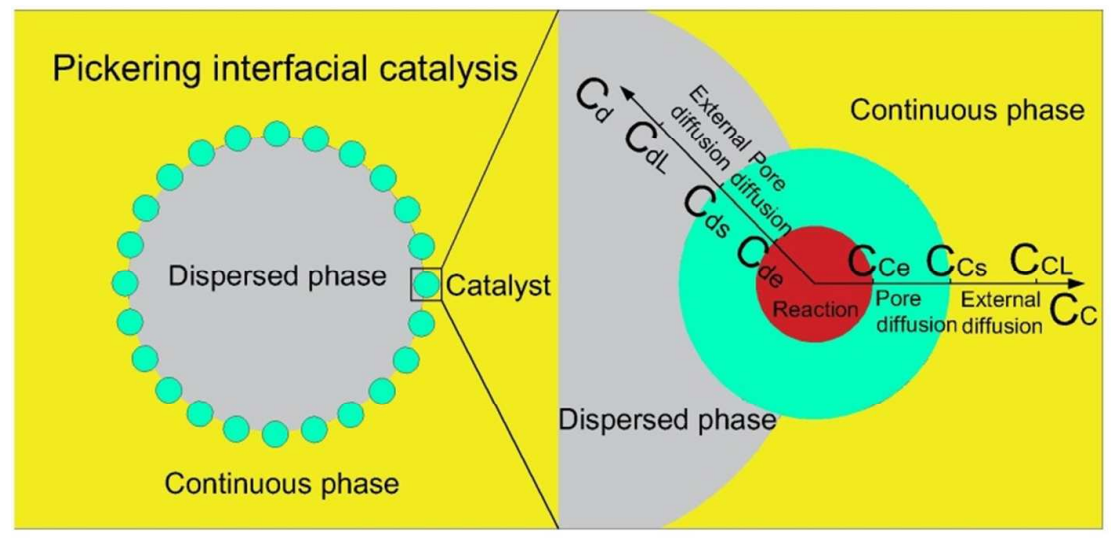


Figure 10. Schematic of interfacial catalysis route in a Pickering emulsion.

For external diffusion, the mass transport rate of a solute (dispersed and continuous phase) can be expressed in terms of overall transport coefficients as follows:

$$
\begin{aligned}
& \text { dispersed phase: } N_{d}=K_{d} a_{d}\left(C_{d L}-C_{d S}\right) \\
& \text { continuous phase: } N_{c}=K_{c} a_{c}\left(C_{c L}-C_{c S}\right)
\end{aligned}
$$

where $K_{d}$ and $K_{c}$ are the overall mass transport coefficients of the dispersed and continuous phase respectively and $a_{d}$ and $a_{c}$ denote the overall mass transport area of the dispersed and continuous phase which can be expressed as the interfacial contact area of the catalyst particles with the dispersed and continuous phase. $\mathrm{C}_{\mathrm{dL}}$ and $\mathrm{C}_{\mathrm{cL}}$ are the concentrations of dispersed and continuous phase respectively. $\mathrm{C}_{\mathrm{dS}}$ and $\mathrm{C}_{\mathrm{cS}}$ are the concentrations of dispersed and continuous phase at the catalyst particle exterior surface respectively. $\mathrm{N}_{\mathrm{d}}$ and $\mathrm{N}_{\mathrm{c}}$ are the diffusion fluxes of dispersed and continuous phase respectively. The larger the overall mass transport area, the smaller the external diffusion resistance. It is evident that both $a_{d}$ and $a_{c}$ mainly rely on the emulsion droplet size.

In the case of chemical reaction in a porous catalyst, pore diffusion is coupled with interfacial reaction. The diffusion reaction equations can be expressed as follows:

$$
\begin{aligned}
& \text { dispersed phase: } D_{e d} \frac{d^{2} C_{d}}{d r^{2}}+D_{e d} \frac{2}{r} \frac{d C_{d}}{d r}=R_{d} \\
& \text { continuous phase: } D_{e c} \frac{d^{2} C_{c}}{d r^{2}}+D_{e c} \frac{2}{r} \frac{d C_{c}}{d r}=R_{c}
\end{aligned}
$$

where $D_{e d}$ and $D_{e c}$ denote effective diffusion coefficients of the dispersed and continuous phase respectively, $\frac{d C_{d}}{d r}$ and $\frac{d C_{c}}{d r}$ represent the derivative of the dispersed 
and continuous phase concentration with spatial distance, $\frac{d^{2} C_{d}}{d r^{2}}$ and $\frac{d^{2} C_{c}}{d r^{2}}$ denote the second derivative of the dispersed and continuous phase concentration with spatial distance and $R_{d}$ and $R_{c}$ signify the chemical reaction rate of the dispersed and continuous phase respectively. The larger the effective diffusion coefficients, the smaller the pore diffusion resistance. It is apparent that $D_{e d}$ and $D_{e c}$ strongly rely on the porosity character of the catalyst.

As for interfacial reaction, epoxidation involves formation of 6-coordinated Ti-OOH intermediates formed by the adsorption of hydrogen peroxide on titanium centers, and reaction of these intermediates with alkenes to give epoxides ${ }^{38,39}$. It is reported that interfacial reaction is several orders of magnitude faster than external diffusion and pore diffusion. Accordingly, the latter determines inter-phase mass transfer and catalyst catalytic activity in Pickering interfacial catalysis. For external diffusion, the overall mass transport areas $a_{d}$ and $a_{c}$ determine external diffusion resistance. As can be seen in Figure 11, the average emulsion droplet diameter decreases progressively with an increase in the degree of hydrophobization of HMDSO/HTS- 1 particles from around $38 \mu \mathrm{m}$ to $12 \mu \mathrm{m}$ for $\mathrm{x}=0.1$ to 0.5 , which is confirmed by optical micrographs of the W/O emulsion droplets shown in the inset. In parallel, the decrease of average droplet diameter is accompanied by an increase in the interfacial contact area and a decrease of the coverage of droplet interfaces by emulsifier particles (calculation is provided in Supporting Information), ${ }^{40}$ shown in Figure S7. High inter-phase contact area compromises external diffusion resistance at the oil-water interface and leads to an enhancement in the catalysis efficiency ${ }^{41}$. From this analysis, we would expect a continuous enhancement in catalytic activity with the degree of hydrophobization. However, the experimental results revealed a parabolic distribution of the TOF values with the 0.2-HMDSO/HTS-1 particle sample attaining the maximum. This observation suggests that in addition to external mass transfer, which is governed by the emulsion droplet size, pore mass transfer is also important for the catalytic activity of HTS-1 particles. 


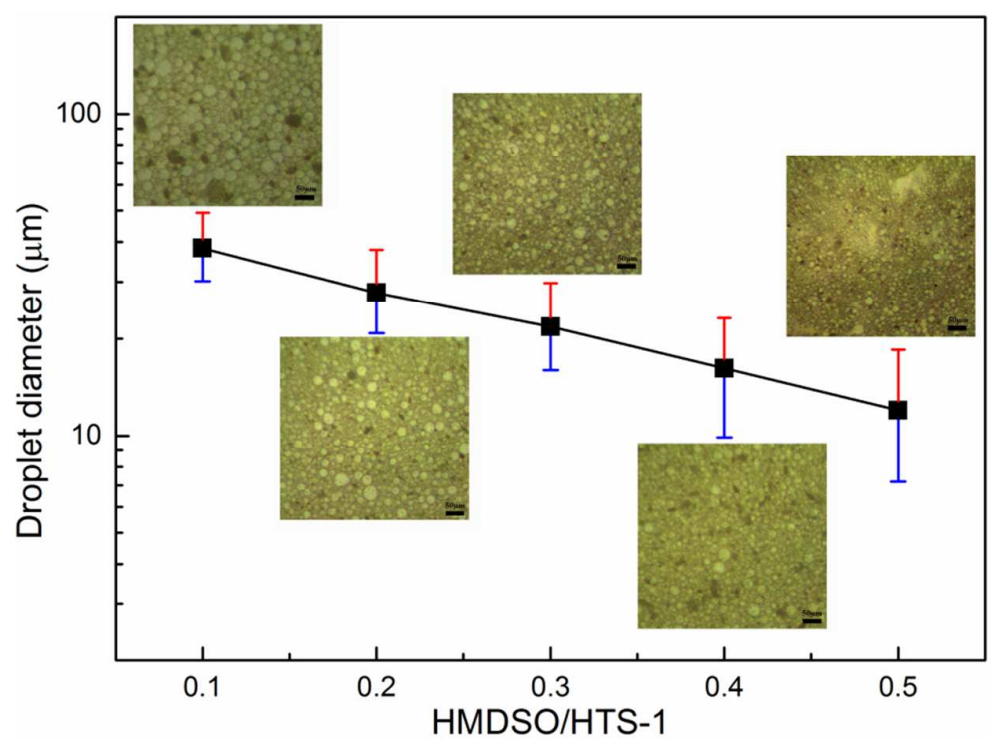

Figure 11. Z-average diameter of droplets in W/O emulsions stabilised by different modified HTS-1 particles and their corresponding micrographs.

For pore diffusion, the effective diffusion coefficients $D_{e d}$ and $D_{e c}$, which strongly depend on catalyst pore character, determine the pore diffusion resistance. $\mathrm{N}_{2}$ adsorption-desorption isotherms were measured at $77 \mathrm{~K}$ to analyze catalyst pore character. Each sample shows a typical type IV isotherm with an H4 hysteresis loop (see Figure S8), which indicates that mesoporous structure is maintained after hydrophobic modification by HMDSO. The results for all the $\mathrm{x}$-HMDSO/HTS-1 samples are summarized in Table 1 . In summary, the pore diameter becomes slightly narrower on increasing the degree of silanisation, whilst the BET surface area and total volume both decrease progressively. In the silylation process, hydroxyl groups are substituted by trimethylsilyl groups. It is noteworthy that the micropore area and micropore volume are independent of the degree of hydrophobization whereas the mesopore area and mesopore volume decrease gradually. Intra-crystalline voids and grooves on HTS-1 zeolite may facilitate the entry of HMDSO molecules into pores and the hydrophobic modification of pore surfaces. The decrease of the surface area, total pore volume and pore diameter may generate inner diffusion resistance and reduce the accessibility of 1-hexene and cyclohexene to the Ti peroxo species 
(Ti-OOH) generated on particles. This limited accessibility upon hydrophobization ascribed to partial blockage of mesopores may be the origin of the decreased TOF values as $\mathrm{x}$ varies from 0.2 to 0.5 .

Table 1. Characteristics of the modified HTS-1 particles derived from $\mathrm{N}_{2}$ isotherms.

\begin{tabular}{|c|c|c|c|c|c|c|c|}
\hline Sample & $\begin{array}{l}\mathrm{S}_{\mathrm{BET}}^{\mathrm{a}} / \\
\mathrm{m}^{2} / \mathrm{g}\end{array}$ & $\begin{array}{c}\mathrm{S}_{\text {micro }}^{\mathrm{c}} / \\
\mathrm{m}^{2} / \mathrm{g}\end{array}$ & $\begin{array}{l}\mathrm{S}_{\text {meso }} \mathrm{d} / \\
\mathrm{m}^{2} / \mathrm{g}\end{array}$ & $\begin{array}{l}\mathrm{V}_{\text {tot }}^{\mathrm{b}} / \\
\mathrm{cm}^{3} / \mathrm{g}\end{array}$ & $\begin{array}{l}\mathrm{V}_{\text {micro }} \mathrm{c} / \\
\mathrm{cm}^{3} / \mathrm{g}\end{array}$ & $\begin{array}{l}\mathrm{V}_{\text {meso }} \mathrm{d} / \\
\mathrm{cm}^{3} / \mathrm{g}\end{array}$ & $\begin{array}{r}\mathrm{D}_{\text {Pore }} / \\
\mathrm{nm}\end{array}$ \\
\hline 0.1-HMDSO/HTS-1 & 420 & 257 & 164 & 0.42 & 0.12 & 0.30 & 11.6 \\
\hline 0.2-HMDSO/HTS-1 & 402 & 256 & 146 & 0.39 & 0.11 & 0.28 & 11.4 \\
\hline 0.3-HMDSO/HTS-1 & 382 & 254 & 128 & 0.35 & 0.11 & 0.24 & 10.9 \\
\hline 0.4-HMDSO/HTS-1 & 368 & 254 & 114 & 0.33 & 0.11 & 0.22 & 10.4 \\
\hline 0.5-HMDSO/HTS-1 & 348 & 253 & 95 & 0.29 & 0.10 & 0.19 & 9.8 \\
\hline
\end{tabular}

${ }^{a} \mathrm{~S}_{\mathrm{BET}}$ is the specific surface area, ${ }^{b} \mathrm{~V}_{\text {tot }}$ is measured for adsorption pore volume at $\mathrm{P} / \mathrm{P}_{0}$ $=0.990,{ }^{\mathrm{c}} \mathrm{S}_{\text {micro }}$ and $\mathrm{V}_{\text {micro }}$ are calculated by t-plot method, ${ }^{\mathrm{d}} \mathrm{S}_{\text {meso }}=\mathrm{S}_{\mathrm{BET}}-\mathrm{S}_{\text {micro }}, \mathrm{V}_{\text {meso }}$ $=\mathrm{V}_{\mathrm{BET}}-\mathrm{V}_{\text {micro }}$.

Based on the above discussion, it is evident that hydrophobic modification relates to emulsion droplet and catalyst pore size changes, which synergistically result in the parabolic TOF distribution. Silane modification of particles results in decreased emulsion droplet size, which benefits the external diffusion and accessibility of 1-hexene and cyclohexene into catalyst pores, and accordingly enhances catalyst catalytic activity. Decreased catalyst pore size and pore volume caused by silane modification raises pore diffusion resistance and impedes the accessibility of 1-hexene and cyclohexene to Ti-OOH active sites, hindering catalyst catalytic activity. From $\mathrm{x}=0.1$ to 0.2 , favorable external diffusion dominates leading to an increased TOF value whereas detrimental pore diffusion takes over and results in decreasing TOF values for $\mathrm{x}=0.2$ to 0.5 .

Finally, the re-usability of the most effective 0.2-HMDSO/HTS-1 particles was 
assessed in the epoxidation of 1-hexene and cyclohexene with aqueous $\mathrm{H}_{2} \mathrm{O}_{2}$ under Pickering interfacial catalysis conditions. After each reaction, the particles were separated by centrifugation, washed with distilled water and dried at $353 \mathrm{~K}$ in a vacuum oven overnight. The recovered catalyst particles were used in a fresh Pickering emulsion reaction mixture. As shown in Table S2, these particles could be successfully recycled for five times without a significant decrease in catalytic activity for epoxidation reactions. These results demonstrate that $0.2-\mathrm{HMDSO} / \mathrm{HTS}-1$ catalyst is a very active and stable Pickering interfacial catalyst for 1-hexene and cyclohexene epoxidation reactions.

\section{Conclusions}

Hydrophobically-modified hollow TS-1 particles were prepared for the fabrication of a Pickering interfacial catalysis system to realize solvent-free alkene epoxidation without addition of solvent. The epoxidation results in W/O emulsions revealed a parabolic distribution of TOF value with the degree of silanisation of particles with 0.2-HMDSO/HTS-1 particles attaining the maximum value of $20.6 \mathrm{~h}^{-1}$ for 1-hexene epoxidation and $8.1 \mathrm{~h}^{-1}$ for cyclohexene epoxidation. Progressive silanisation of particles leads to a reduction in the average emulsion droplet size which benefits external diffusion. This, together with the decreased catalyst pore size and pore volume which impede pore diffusion, result in the parabolic TOF distribution. The 0.2-HMDSO/HTS-1 particles showed excellent re-usability for more than five cycles.

\section{Acknowledgements}

We gratefully acknowledge financial support by the National Basic Research Program of China (973 Program) (Grant No: 2012CB720302) and the National Key Research and Development Program of China (2016YFF0102503). 


\section{References}

(1) Xiao, F. S.; Wang, L. F.; Yin, C. Y.; Lin, K. F.; Di, Y.; Li, J. X.; Xu, R. R.; Su, D. S.; Schlögl, R.; Yokoi, T.; Tatsumi, T. Catalytic properties of hierarchical mesoporous zeolites templated with a mixture of small organic ammonium salts and mesoscale cationic polymers. Angew. Chem. Int. Ed. 2006, 118, 3162-3165.

(2) Lin, W. G.; Wan, M. M.; Zhou, Y.; Gu, H. C.; Zhou, S. L.; Zhu, J. H. Novel selective catalyst derived from uniform clustered $\mathrm{NaY}$ zeolite microspheres. J. Mater. Chem. A 2013, 1, 6849-6857.

(3) Liu, X. H.; Yang, C. Y.; Wang, Y. Q.; Guo, Y. L.; Guo, Y.; Lu, G. Z. Effect of the diatomite pretreatment on the catalytic performance of TS-1/diatomite for toluene hydroxylation by $\mathrm{H}_{2} \mathrm{O}_{2}$ in fixed-bed reactor. Chem. Eng. J. 2014, 243, 192-196.

(4) Lu, X. Q.; Zhou, W. J.; Wu, H. H.; Liebens, A.; Wu, P. Selective synthesis of ethylene oxide through liquid-phase epoxidation of ethylene with titanosilicate $/ \mathrm{H}_{2} \mathrm{O}_{2}$ catalytic systems. Appl. Catal. A: Gen. 2016, 515, 51-59.

(5) Xu, Y. Y.; Yang, Q. S.; Li, Z. H.; Gao, L. Y.; Zhang, D. S.; Wang, S. F.; Zhao, X.

Q.; Wang, Y. J. Ammoximation of cyclohexanone to cyclohexanone oxime using ammonium chloride as nitrogen source. Chem. Eng. Sci. 2016, 152, 717-723.

(6) Xu, L.; Lv, G. J.; Li, H.; Shen, Y.; Cai, W. F.; Wang, F. M.; Zhang, X. B. Surface-modified TS-1 with enhanced activity for cyclohexanone ammoximation in a Pickering emulsion and increased stability in hot aqueous ammonia. $R S C A d v . \mathbf{2 0 1 5}, 5$, $62652-62658$.

(7) Fan, W. B.; Wu, P.; Tatsumi, T. Unique solvent effect of microporous crystalline titanosilicates in the oxidation of 1-hexene and cyclohexene. J. Catal. 2008, 256, $62-73$.

(8) Li, H.; Xu, B.; Deng, B. W.; Yan, X. M.; Zheng, Y. C. Epoxidation of 1-hexene with hydrogen peroxide over nitrogen-incorporated TS-1 zeolite. Catal. Commun. 2014, 46, 224-227.

(9) Tekla, J.; Tarach, K. A.; Olejniczak, Z.; Girman, V.; Gora-Marek, K. Effective hierarchization of TS-1 and its catalytic performance in cyclohexene epoxidation. Micropor. Mesopor. Mat. 2016, 233, 16-25. 
(10) Pera-Titus, M.; Leclercq, L.; Clacens, J. M.; De Campo, F.; Nardello-Rataj, V. Pickering interfacial catalysis for biphasic systems: from emulsion design to green reactions. Angew. Chem. Int. Ed. 2015, 54, 2006-2021.

(11) Shi, H.; Fan, Z. Y.; Ponsinet, V.; Sellier, R.; Liu, H. L.; Pera-Titus, M.; Clacens, J. M. Glycerol/Dodecanol double Pickering emulsions stabilized by polystyrene-grafted silica nanoparticles for interfacial catalysis. ChemCatChem 2015, 7, 3229-3233.

(12) Cole-Hamilton, D. J. Janus catalysts direct nanoparticle reactivity. Science 2010, $327,41-42$.

(13) Ungureanu, S.; Deleuze, H.; Sanchez, C.; I. Popa, M.; Backov, R. First Pd@Organo-Si (HIPE) open-cell hybrid monoliths generation offering cycling heck catalysis reactions. Chem. Mater. 2008, 20, 6494-6500.

(14) Fan, Z. Y.; Tay, A.; Pera-Titus, M.; Zhou, W. J.; Benhabbari, S.; Feng, X. S.; Malcouronne, G.; Bonneviot, L.; De Campo, F.; Wang, L. M.; Clacens, J. M. Pickering Interfacial Catalysts for solvent-free biomass transformation: Physicochemical behavior of non-aqueous emulsions. J. Colloid. Interface Sci. 2014, $427,80-90$.

(15) Ikeda, S.; Nur, H.; Sawadaishi, T.; Ijiro, K.; Shimomura, M.; Ohtani, B. Direct observation of bimodal amphiphilic surface structures of zeolite particles for a novel liquid-liquid phase boundary catalysis. Langmuir, 2001, 17, 7976-7979.

(16) Liu, H. F.; Zhang, Z. M.; Yang, H. Q.; Cheng, F. Q.; Du, Z. P. Recycling nanoparticle catalysts without separation based on a Pickering emulsion/organic biphasic system. ChemSusChem, 2014, 7, 1888-1900.

(17) Xue, F.; Zhang, Y. B.; Zhang, F. W.; Ren, X. M.; Yang H. Q. Tuning the interfacial activity of mesoporous silicas for biphasic interface catalysis reactions. ACS Appl. Mater. Interfaces, 2017, 9, 8403-8412.

(18) Yang, Y. L.; Zhou, W. J.; Liebens, A.; Clacens, J. M.; Pera-Titus, M.; Wu, P. Amphiphilic titanosilicates as Pickering interfacial catalysts for liquid-phase oxidation reactions. J. Phys. Chem. C 2015, 119, 25377-25384.

(19) Pérez-Ramírez, J.; Christensen, C. H.; Egeblad, K.; Christensen, C. H.; Groen, J. C. Hierarchical zeolites: enhanced utilisation of microporous crystals in catalysis by 
advances in materials design. Chem. Soc. Rev. 2008, 37, 2530-2542.

(20) Louis, B.; Ocampo, F.; Yun, H. S.; Tessonnier, J. P.; Maciel Pereira, M. Hierarchical pore ZSM-5 zeolite structures: From micro- to macro-engineering of structured catalysts. Chem. Eng. J. 2010, 161, 397-402.

(21) Chal, R.; Gérardin, C.; Bulut, M.; van Donk, S. Overview and industrial assessment of synthesis strategies towards zeolites with mesopores. ChemCatChem 2011, 3, 67-81.

(22) Wu, X. X.; Wang, Y. Q.; Zhang, T.; Wang, S. H.; Yao, P. X.; Feng, W. P.; Xu, J. Effect of TS-1 treatment by tetrapropyl ammonium hydroxide on cyclohexanone ammoximation. Catal. Commun. 2014, 50, 59-62.

(23) Yang, H. Q.; Zhou, T.; Zhang, W. J. A strategy for separating and recycling solid catalysts based on the pH-triggered Pickering-emulsion inversion. Angew. Chem. Int. Ed. 2013, 125, 7603-7607.

(24) Wang, Y. R.; Lin, M.; Tuel, A. Hollow TS-1 crystals formed via a dissolution-recrystallization process. Micropor. Mesopor. Mat. 2007, 102, 80-85.

(25) Wang, X. B.; Zhang, X. F.; Wang, Y.; Liu, H. O.; Wang, J. Q.; Qiu, J. S.; Ho, H. L.; Han, W.; Yeung, K. L. Preparation and performance of $\mathrm{TS}-1 / \mathrm{SiO}_{2}$ egg-shell catalysts. Chem. Eng. J. 2011, 175, 408-416.

(26) Lv, G. J.; Wang, F. M.; Zhang, X. B. Easily recoverable micron-sized silica-walled TS-1 colloidosomes: Preparation and application as liquid-phase alkene epoxidation catalysts. Appl. Catal. A: Gen. 2017, 547, 191-198.

(27) Wang, X. B.; Zhang, X. F.; Liu, H. O.; Yeung, K. L.; Wang, J. Q. Preparation of titanium silicalite-1 catalytic films and application as catalytic membrane reactors. Chem. Eng. J. 2010, 156, 562-570.

(28) Liu, C.; Huang, J. L.; Sun, D. H.; Zhou, Y.; Jing, X. L.; Du, M. M.; Wang, H. T.; Li, Q. B. Anatase type extra-framework titanium in TS-1: a vital factor influencing the catalytic activity toward styrene epoxidation. Appl. Catal. A: Gen. 2013, 459, 1-7. (29) Cheneviere, Y.; Chieux, F.; Caps, V.; Tuel, A. Synthesis and catalytic properties of TS-1 with mesoporous/microporous hierarchical structures obtained in the presence of amphiphilic organosilanes. J. Catal. 2010, 269, 161-168. 
(30) Du, Q.; Guo, Y. P.; Duan, H. N.; Li, H.; Chen, Y. J.; Liu, H. Z. Synthesis of hierarchical TS-1 zeolite via a novel three-step crystallization method and its excellent catalytic performance in oxidative desulfurization. Fuel 2017, 188, 232-238.

(31) Ricchiardi, G.; Damin, A.; Bordiga, S.; Lamberti, C.; Spanò, G.; Rivetti, F.; Zecchina, A. Vibrational structure of titanium silicate catalysts. A spectroscopic and theoretical study. J. Am. Chem. Soc. 2001, 123, 11409-11419.

(32) Binks, B. P.; Lumsdon, S. O. Influence of particle wettability on the type and stability of surfactant-free emulsions. Langmuir 2000, 16, 8622-8631.

(33) Finkle, P.; Draper, H. D.; Hildebrand, J. H. The theory of emulsification. J. Am. Chem. Soc. 1923, 45, 2780.

(34) Pickering, S. U. Emulsions, J. Chem. Soc. 1907, 91, 2001-2021.

(35) Bormashenko, E. Y. Physics of Wetting: Phenomena and Applications of Fluids on Surfaces, Walter de Gruyter GmbH, 2017.

(36) Zapata, P. A.; Faria, J.; Ruiz, M. P.; Resasco, D. E. Condensation/hydrogenation of biomass-derived oxygenates in water/oil emulsions stabilized by nanohybrid catalysts. Top. Catal. 2012, 55, 38-52.

(37) Fogler, H. S. Elements of chemical reaction engineering, Prentice-Hall International, New Jersey, 2009.

(38) Wang, L. L.; Xiong, G.; Su, J.; Li, P.; Guo, H. C. In situ UV raman spectroscopic study on the reaction intermediates for propylene epoxidation on TS-1. J. Phys. Chem. C 2012, 116, 9122-9131.

(39) Kwon, S.; Schweitzer, N. M.; Park, S. Y.; Stair, P. C.; Snurr, R. Q. A kinetic study of vapor-phase cyclohexene epoxidation by $\mathrm{H}_{2} \mathrm{O}_{2}$ over mesoporous TS-1. J. Catal. 2015, 326, 107-115.

(40) Gautier, F.; Destribats, M.; Perrier-Cornet, R.; Dechézelles J. F.; Héroguez, V.; Ravaine, S.; Leal-Calderon, F.; Schmitt, V. Pickering emulsions with stimulable particles: from highly-to weakly-covered interfaces. Phys. Chem. Chem. Phys. 2007, 9, 6455-6462. 
(41) Zhang, M.; Wei, L. J.; Chen, H.; Du, Z. P.; Binks, B. P.; Yang, H. Q. Compartmentalized droplets for continuous flow liquid-liquid interface catalysis. $J$. Am. Chem. Soc. 2016, 138, 10173-10183. 